\title{
Co-Administered Vitamin E Isoforms d- $\alpha$-tocopherol and d- $\delta$-tocotrienol Rich Fraction Promote Regeneration of Skeletal Muscle in Diabetics
}

\author{
Bijo Elsy", Aijaz Ahmed Khan ${ }^{1,}$, , Veena Maheshwari ${ }^{2}$ \\ ${ }^{1}$ Department of Anatomy, JN Medical College, Aligarh Muslim University, Aligarh, India \\ ${ }^{2}$ Department of Pathology, JN Medical College, Aligarh Muslim University, Aligarh, India \\ Email address: \\ bijobaby22@yahoo.com (B. Elsy), aijazahmedkhan7@live.com (A. A. Khan), vegovil@gmail.com (V. Maheshwari) \\ ${ }^{*}$ Corresponding author
}

To cite this article:

Bijo Elsy, Aijaz Ahmed Khan, Veena Maheshwari. Co-Administered Vitamin E Isoforms d- $\alpha$-Tocopherol and d- $\delta$-Tocotrienol Rich Fraction Promote Regeneration of Skeletal Muscle in Diabetics. International Journal of Nutrition and Food Sciences. Vol. 7, No. 2, 2018 , pp. 47-55. doi: $10.11648 /$ j.ijnfs.20180702.12

Received: January 22, 2018; Accepted: February 5, 2018; Published: February 27, 2018

\begin{abstract}
In diabetes the structural and functional recovery of skeletal muscle is impaired due to persistent hyperglycemiainduced oxidative stress. Vitamin E is known to be essential antioxidant for maintains the skeletal muscle homeostasis thus preventing oxidative damages. This study is designed to explore the effect of $d$ - $\alpha$-tocopherol and $d-\delta$-tocotrienol rich fraction (d- $\delta$-TRF) on crushed muscle regeneration in both healthy and diabetic rats. Diabetes was induced through single subcutaneous injection of aqueous alloxan at the dose of $100 \mathrm{mg} / \mathrm{kg}$. Twenty four albino rats were divided into four groups; healthy control, diabetic control, healthy treated and diabetic treated. Treated groups received $100 \mathrm{mg} / \mathrm{kg}$ of d- $\alpha$-tocopherol and d- $\delta$-TRF each, orally, daily for three weeks. Through a horizontal mid-thigh skin incision and splitting of the fascia gluteus maximus was approached and crushed with Kocher's forceps. Skin wound was closed with an absorbable suture. The crush-induced degenerative and regenerative changes in the muscle were studied by assessing the histological features, histomorphological measurements and biochemical analyses at the end of $3^{\text {rd }}$ weeks. One- way 'ANOVA' and Student's $t$-test were used for statistical analysis. All results revealed that the vitamin E isoforms have potency to maintain glycemic level, improve the antioxidant capacity and hasten the process of regeneration, revascularization, reinnervation and connective tissue remodeling in skeletal muscle after crush injury. It is therefore, concluded that the vitamin E isoforms are useful nutritional supplements for skeletal muscle functional and structural recovery in both healthy and diabetics.
\end{abstract}

Keywords: Antioxidant, Crush-Injury, d- $\alpha$-tocopherol, $d-\delta$-tocotrienol Rich Fraction, Diabetes, Rats, Skeletal Muscle

\section{Introduction}

Skeletal muscle regenerative capacity involves the number, activation, proliferation and differentiation capacities of satellite cells as well as environment and oxidative stress $[1,2]$.

Marked muscle atrophy is a characteristic feature of uncontrolled diabetes [3]. Oxidative stress severely impairs plasma membrane repair which is a fundamental cellular activity of the skeletal myocyte and failure to repair of the membrane may result into cell death by necrosis. Vitamin E is required for plasma membrane repair in skeletal myocytes $[4,5]$ and also it protects the muscle from diabetes-induced oxidative damage in rats [3]. All above-mentioned studies are mainly based on the $\alpha$-tocopherol isoform.

Another study [1] revealed that only low doses of tocotrienol rich fraction (TRF) promotes the proliferation capacity of the myoblasts but its high doses $(>200 \mu \mathrm{g} / \mathrm{ml})$ has negative effect on proliferation capacity and is cytotoxic to the myoblasts. Since TRF contain different vitamin E isoforms among which the $\gamma$-tocotrienol is the major constituent [6].

Our previous studies $[7,8]$ revealed that the individual supplementation of $\mathrm{d}-\alpha$-tocopherol and $\mathrm{d}-\delta$-TRF helped to accelerate the skeletal muscle regeneration after crushed injuries. Therefore this present study is designed to assess the effect of co-administration of these compounds on muscle repair in both 
healthy and diabetic rats by using functional, histopathological, histomorphological and biochemical parameters.

\section{Materials and Methods}

After the approval by Institutional Animal Ethical Committee (No. 8937/2014), twenty four albino rats of either sex each weighing 230-320g were obtained from central animal house of JN medical college, AMU, Aligarh.

This present study followed the same method of animal care, induction of diabetes and monitoring of blood glucose level as described in our previous study [9].

\subsection{Experimental Groups, Route and Dosage of Treatment}

Animals were divided into four groups having six rats in each group: (1) healthy control- HC; (2) diabetic controlDC; (3) healthy $d-\alpha$-tocopherol and $d-\delta$-TRF treated- HXT and (4) diabetic $d-\alpha$-tocopherol and $d-\delta$-TRF treated- DXT $(100 \mathrm{mg} / \mathrm{kg}$ of $\mathrm{d}-\alpha$-tocopherol and $\mathrm{d}-\delta$-TRF each, orally, daily for three weeks). $d-\alpha$-Tocopherol Myra e capsule [Vitamin E] manufactured by PT Daya- Baria laboratoria Tbk, Indonesia; Imported and packed by United laboratories, Philippines. Unique E Tocotrienol, Tocopherol free $[90 \% \delta$ and $10 \% \gamma$ tocotrienols] AC Grace Company, TX 75755, USA).

\subsection{Surgical Procedure}

Surgical procedure (Figure 1), sample collection and fixation of tissues were followed the methods as described in our previous studies [7-9].

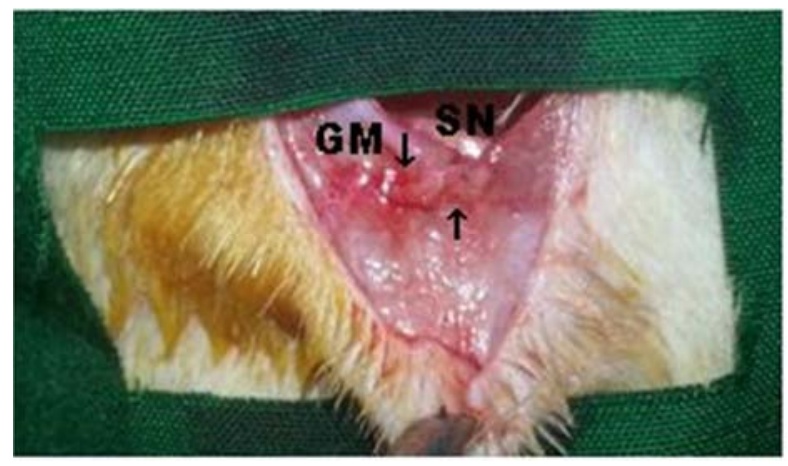

Figure 1. Photograph showing crushed parts of the Gluteus Maximus muscle.

Note: GM- Gluteus Maximus muscle, SN- Sciatic Nerve and Arrow $(\rightarrow)$ pointing the crushed parts of GM.

\subsection{Histopathology and Histomorphology}

Fixed tissue samples were processed for light microscopic studies. The $5 \mu \mathrm{m}$ thick paraffin sections were stained with Haematoxylin \& Eosin (H \& E), Aldehyde Fuchsin with Fast Green (AF with FG) and PicroSirus Red with Fast Green (PSR with FG).

Methods used in Histomorphological measurements and its calculations were same as described in our previous studies $[7,8]$

\subsection{Biochemical Estimation \& Analysis}

\subsubsection{Lipid profile, Serum Creatinine and Total Protein}

All lipid profiles, serum creatinine and serum total protein content were carried out by using Avantor Benesphera ${ }^{\mathrm{TM}}$ clinical chemistry Analyzer C61.

\subsubsection{Enzymatic Antioxidant}

Serum catalase was assayed by colorimetery as described [10]. The light absorbance of the sample was determined at $620 \mathrm{~nm}$.

\subsubsection{Non-invasive Biomarker (Oxidative Stress Parameter)}

Serum total antioxidant capacity (TAC) was evaluated using ferric reducing antioxidant power (FRAP) assay [11]. The absorbance of sample was measured at $620 \mathrm{~nm}$ using photo colorimeter.

\subsection{Statistical Analysis}

All the data were statistically evaluated and the significance calculated using One-way 'ANOVA' followed by Tukey's test. Student's $t$ - test were used for comparing the initial and final mean body weight of DC and blood glucose level in DXT group before and after treatment. All results were expressed as mean $\pm \mathrm{SD}$ and $\mathrm{P}<0.05$ and $\mathrm{P}<0.0001$ were considered as statistically significant.

\section{Results}

\subsection{Body Weight and Blood Glucose Level}

During the experimental period, typical clinic manifestations of the diabetes such as polyphagia, polydipsia and polyuria were observed in diabetic control rats while these clinical signs were reduced in diabetic treated groups after three weeks co-administration of $\mathrm{d}$ - $\alpha$-tocopherol and $\mathrm{d}$ $\delta$-TRF. Weight and blood glucose levels of all animals in each group were monitored at weekly intervals. Mean body weight in DC was significantly $(\mathrm{P}<0.0001)$ reduced whereas in all other groups it remained stable at the end of study period (Table 1). Mean blood glucose levels of healthy groups (HC \& HXT) remained within normal limits. In DXT the mean blood glucose level was significantly $(\mathrm{P}<0.0001)$ reduced after three weeks treatment while in DC showed $>$ $500 \mathrm{mg} / \mathrm{dl}$ throughout the experimental period (Table 2).

Table 1. Body weights ( $g$ ) of the animals of all groups during the period of study (Mean $\pm S D)$.

\begin{tabular}{lllll}
\hline Groups & Day 0 & Day 7 & Day 14 & Day 21 \\
\hline HC & $270 \pm 35.59$ & $266.67 \pm 15.28$ & $283.33 \pm 20.82$ & $290 \pm 21.60$ \\
DC & $277.5 \pm 25$ & $247.5 \pm 17.08$ & $235 \pm 23.80$ & $227.5 \pm 22.17$ \\
HXT & $270 \pm 29.44$ & $262.5 \pm 17.09$ & $275 \pm 20.82$ & $288.6 \pm 19.87$ \\
DXT & $272 \pm 25.88$ & $252.6 \pm 22.33$ & $268 \pm 23.87$ & $279 \pm 23.02$ \\
\hline
\end{tabular}

Note the mean body weight significantly $(\mathrm{P}<0.0001)$ reduced in $\mathrm{DC}$ at the end of study period while body weight of all other groups remained stable. 
Table 2. Blood glucose ( $\mathrm{mg} / \mathrm{dl})$ level of the animals of all groups during the period of study (Mean $\pm S D$ ).

\begin{tabular}{lllll}
\hline Groups & Day 0 & Day 7 & Day 14 & Day 21 \\
\hline HC & $146 \pm 28.21$ & $124 \pm 19.98$ & $160.67 \pm 18.01$ & $167 \pm 17.06$ \\
DC & $540.25 \pm 47.12$ & $553 \pm 39.42$ & $574.25 \pm 30.20$ & $578 \pm 34.73$ \\
HXT & $145.33 \pm 31.37$ & $153 \pm 32.70$ & $137.5 \pm 20.51$ & $131.33 \pm 29.74$ \\
DXT & $565.4 \pm 39.22$ & $452.5 \pm 36.70$ & $302 \pm 25.51$ & $218 \pm 29.30$ \\
\hline
\end{tabular}

Note that in DXT group the mean blood glucose level was significantly $(\mathrm{P}<0.0001)$ reduced after three weeks treatment while in DC showed $>500$ $\mathrm{mg} / \mathrm{dl}$ throughout the experimental period.

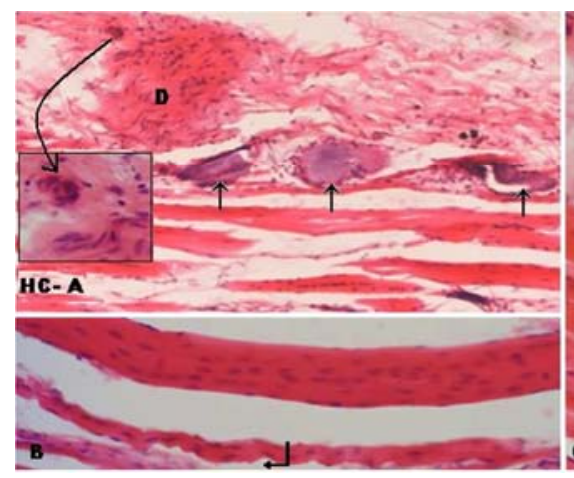

Figure 2. Showing degenerating and necrotic changes in the myofibres of HC group.

\subsection{Microscopic Observations}

\subsubsection{Changes Suggesting Degeneration}

On $3^{\text {rd }}$ week the control groups showed more inflammatory cells, atrophic fibres with hypereosinophilic, hypertrophied and undulated sacrolemma and necrotic fibres with mineralization (Figure $2 \& 3$ ). Other myopathic changes such as swollen, vacuolated, hyalinized, fragmented myofibers and hemorrhages in the myofibers and blood capillaries were noticed in DC (Figure 3). Whereas in treated groups these degenerative and necrotic changes were hardly seen.

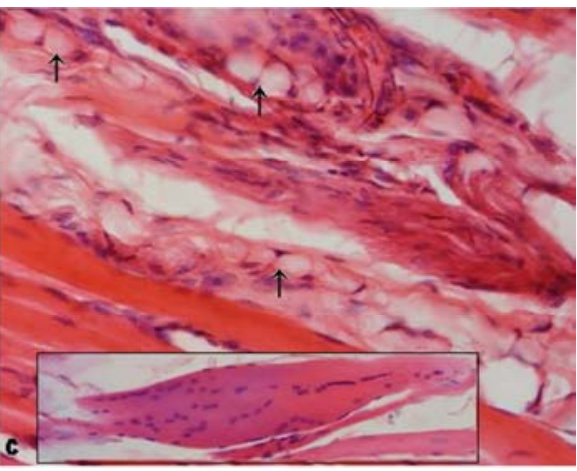
Note: A- $\uparrow:$ Necrotic fibres with mineralization, D: Degenerating fibres at initial magnification x100, inset $\mathbf{S}$ : Multinucleated giant cell at initial magnification
x400.
B-ل: Undulated sacrolemma, C- $\uparrow:$ Fatty depositions in the epimysium, inset: Hypereosinophilic and hypertrophied myofibre at initial magnification of x400. B-」: Undula

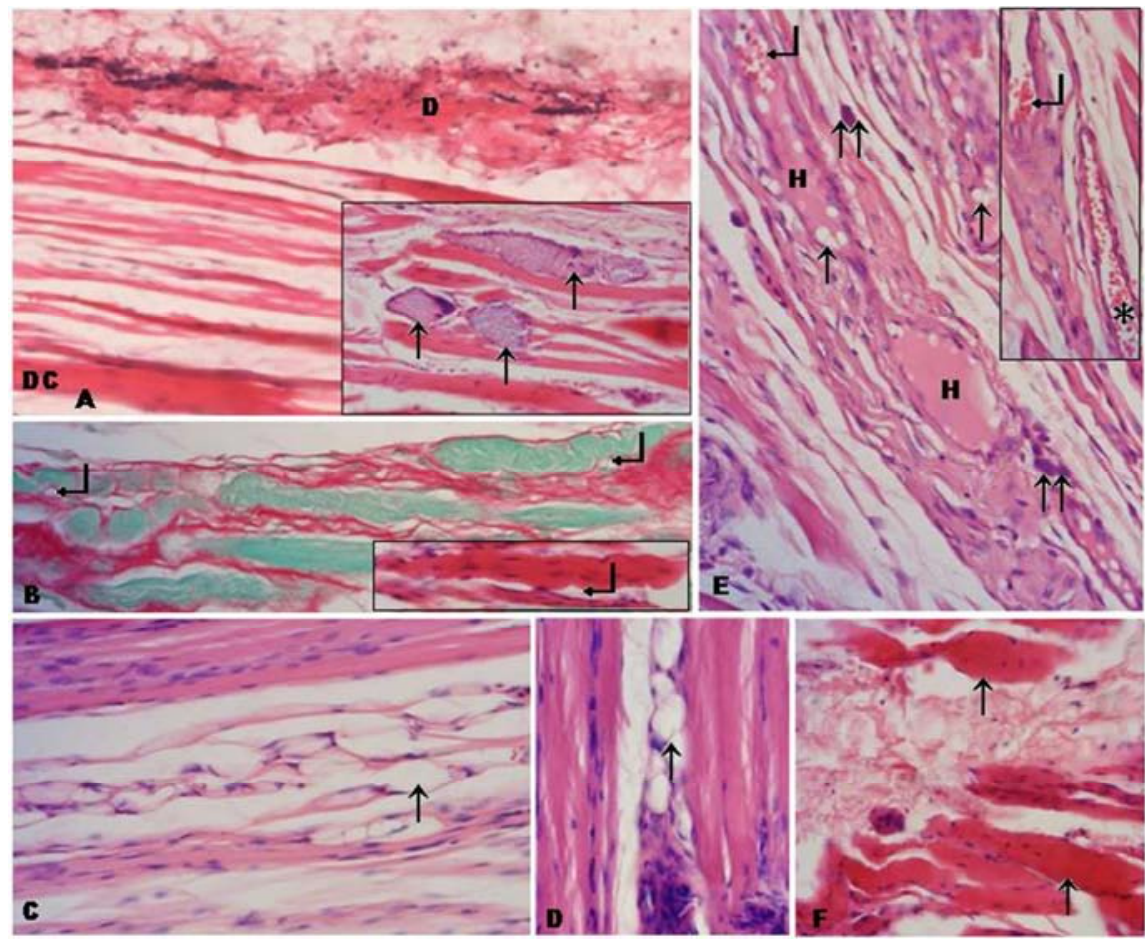

Figure 3. Showing degenerating and necrotic changes in the myofibres in DC group.

Note: A. D: Degenerating fibres, inset $\uparrow:$ Necrotic fibres with mineralization at initial magnification at x100. H \& E stained sections. B.」: Undulated sacrolemma at initial magnification x400. PSR with FG and inset H \& E stain. C-Fatty depositions in the epimysium, D- Fatty depositions in between myofibers, E- $\uparrow$ : Vacuolations, $\uparrow \uparrow:$ Inflammatory cells, H: Hyalinizations, $\lrcorner$ : Hemorrhage in the myofibers and inset, *: Hemorrhage in the blood capillary (inset), F- $\uparrow$ : Hypereosinophilic and hypertrophied myofibre at initial magnification x400. H \& E stain. 


\subsubsection{Changes Suggesting Regeneration}

Only few activated myoblasts were noticed in control groups especially in DC as compared to treated groups (Figure 4). More newly regenerated myofibers (Figure 5), myofibers with central nuclei and split fibres were often noticed in treated groups as compared to control groups (Figure 6).

\subsubsection{Fibrosis and Fatty Connective Tissue}

Quite frequent fatty depositions were found in the epimysium of control groups (Figure 2, 3 \& 9) as compared to treated groups (Figure 9) and in DC these features were also found in the in perimysium and interstitial connective tissues (Figure 3). In control groups fibrosis was marked in all connective tissue coverings as compared to treated groups (Figure 7).

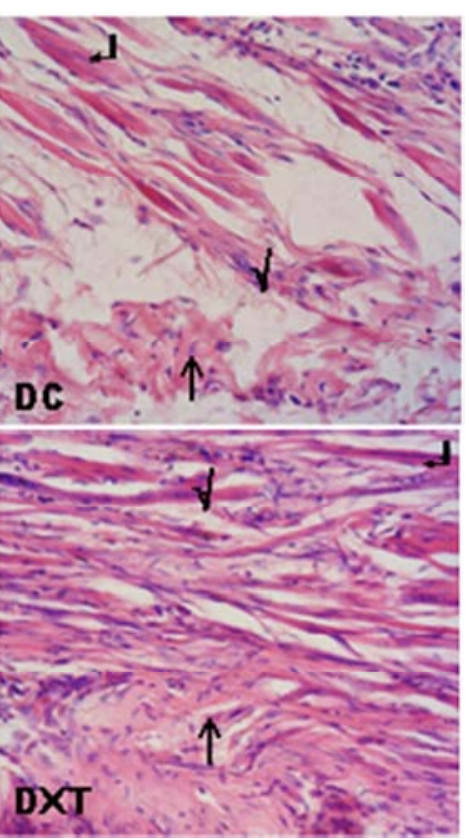

Figure 4. Showing Activated myoblasts in all groups.

Note: $\uparrow:$ Activated satellite cells $(\uparrow)$, Myoblasts with row of vesiculated nuclei $(\sqrt{ })$ and centrally placed myonuclei (\lrcorner$)$, at initial magnification x400. H \& E stain.

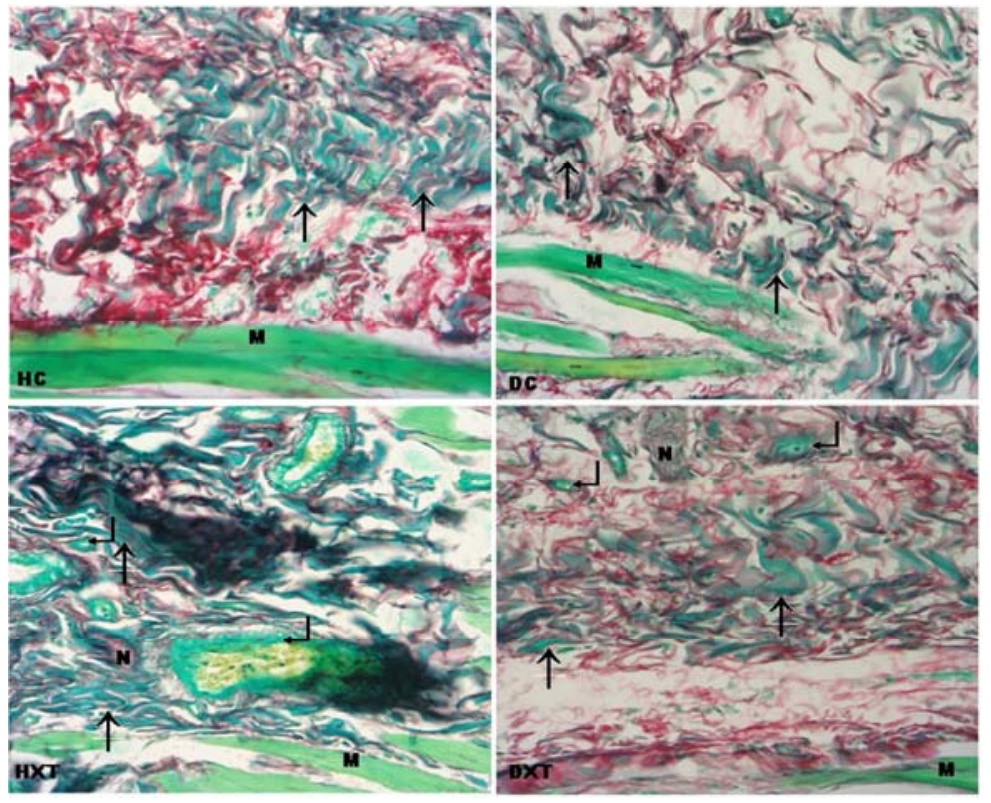

Figure 5. Showing regenerated myofibers in all groups and vascularization and innervations of the regenerated fibres in treated groups.

Note: $\uparrow:$ Regenerated myofibers, M: Normal myofibers and presence of collagen fibres (Red colour) in all groups. N: Nerve bundle, لـ: blood capillaries. In treated groups (HXT \& DXT) nerve bundles and blood capillaries were seen in relation to regenerated myofibers and, at initial magnification x400. PSR with FG stain. 


\subsubsection{Connective Tissue Remodeling and Neovascularization}

Only few thin epimyseal elastin fibres were noticed in $\mathrm{HC}$ while these fibres were almost absent in the connective tissue coverings of DC. In treated groups these fibres were prominent were distributed in all connective tissue coverings (Figure 8). In treated groups more proliferated blood capillaries have shown in the epimysium and at the site of newly regenerated myofibers (Figure 5 \& 9). But these features were less and observed only in the epimysium of control groups (Figure 9).

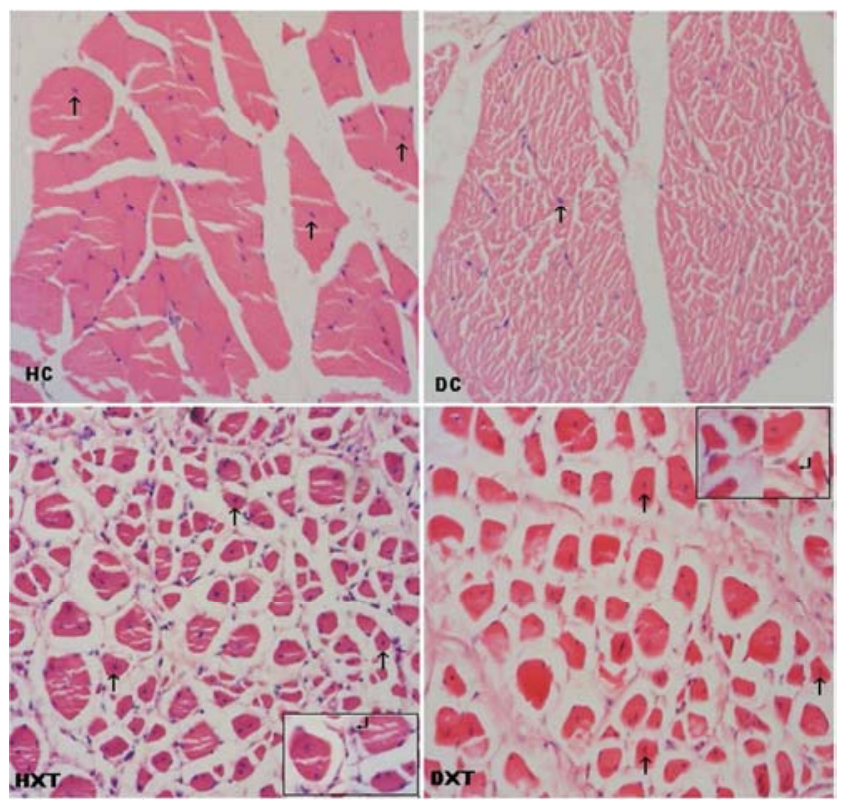

Figure 6. Showing central nuclei in all groups and split fibres in treated groups.

Note: $\uparrow:$ myofibers with central nuclei, in HXT \& DXT inset-لد: split fibres, at initial magnification $\mathrm{x} 400$. $\mathrm{H} \& \mathrm{E}$ stain.

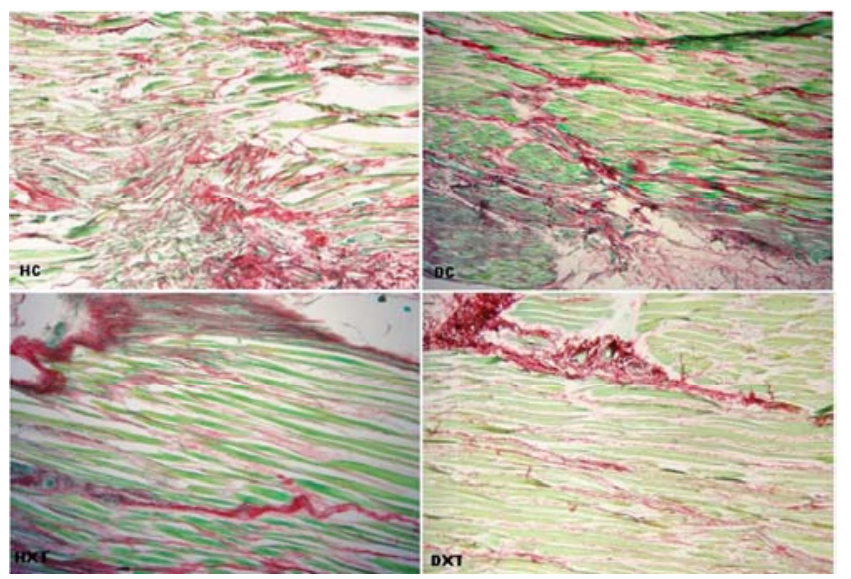

Figure 7. Showing presence and amount of fibrosis in all groups.

Note: Note- more collagen fibres (Red colour) in the all connective tissue layers of control groups but in treated groups these fibres were minimal, at initial magnification $\mathrm{x} 100$. PSR with FG stain.

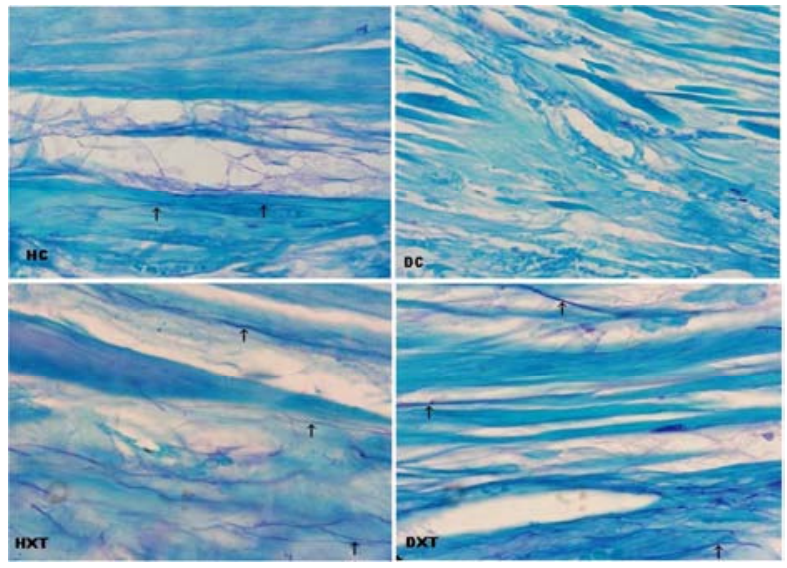

Figure 8. Showing reappearance of Elastin fibres during extracellular matrix remodeling.

Note: $\uparrow:$ Elastin fibres. In HC elastin fibres only in the epimysium, DC these fibres were deficient in all connective tissue coverings but in treated groups have shown reappearance of thin elastin fibres in all connective tissue coverings, at initial magnification $\mathrm{x} 400$. AF with FG stain.

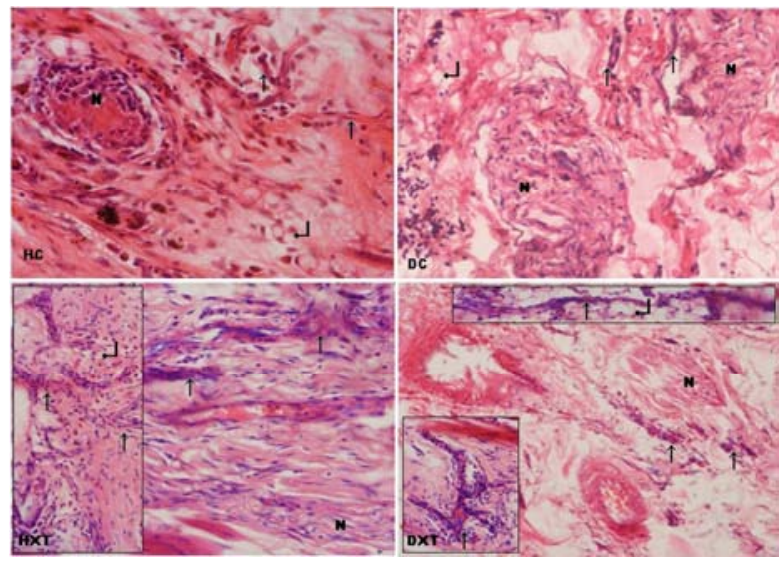

Figure 9. Showing neovascularization of blood capillaries and reinnervations.

Note: $\uparrow:$ Blood capillaries, in treated groups and its inset views have shown numerous proliferated blood capillaries N: Nerve bundle, له: Fat depositions, at initial magnification $\mathrm{x} 400$. $\mathrm{H} \& \mathrm{E}$ stain.

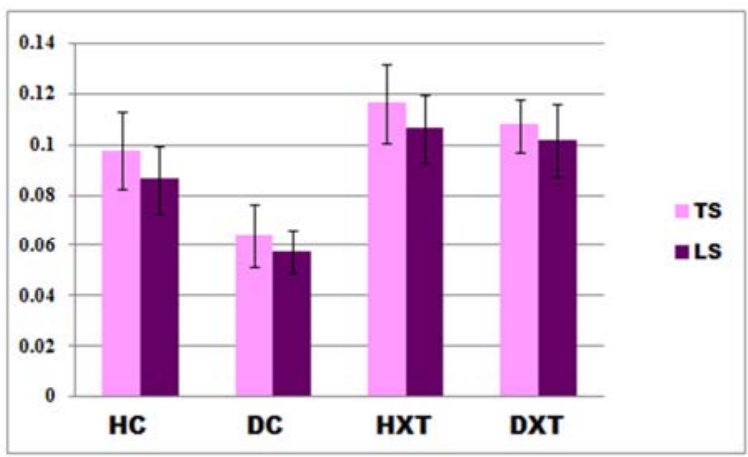

Figure 10. Showing myofibres' width in LS and diameter in TS ((in mm, Mean $\pm S D)$.

Note: In treated groups myofibers' diameter/width were significantly $(\mathrm{P}<0.01)$ increased as compared to control groups. 


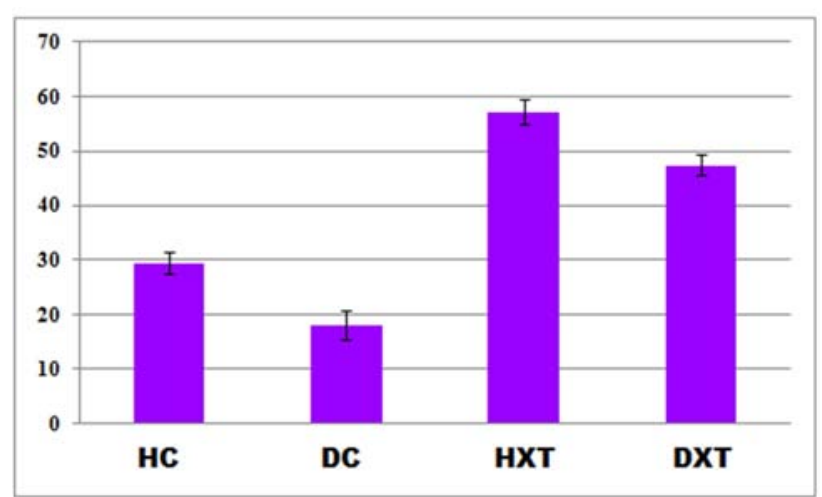

Figure 11. Showing percentage of central nuclei in all groups in muscle TS (Mean $\pm S D)$.

Note: The percentages of central myonuclei in treated group were significantly $(\mathrm{P}<0.01)$ increased as compared to control groups.

\subsubsection{Functional Repair by Reinnervation}

In all groups epimyseal nerve bundles were observed (Figure 9) and these features were also seen in relation to the recently regenerated myofibers of treated groups (Figure $5 \& 9$ ).

\subsection{Histomorphology}

In control groups myofibers width and diameter were significantly $(\mathrm{P}<0.01)$ lesser as compared to treated groups (Figure 10). Percentages of central myonuclei were significantly $(\mathrm{P}<0.01)$ high in treated groups as compared to control groups (Figure 11).

\subsection{Biochemical Analysis on $3^{\text {rd }}$ Week}

\subsubsection{Lipid Profiles}

Total cholesterol (TC), triglycerides (TG), low density lipoprotein (LDL) and very low density lipoprotein (VLDL) in DC were significantly higher $((\mathrm{P}<0.01)$ as compared to DXT. Whereas high density lipoprotein (HDL) in DC showed significantly lower values $(\mathrm{P}<0.01)$ compared to DXT (Table 3) $[12]$.

Table 3. Lipid profiles (Mean $\pm S D$ ) after co-administration of $d$ - $\alpha$-tocopherol and $d-\delta$-TRF.

\begin{tabular}{lllll}
\hline Lipid profiles & & & & \\
\hline Groups & Total cholesterol (mg/d) & HDL (mg/dl) & LDL (mg/dl) & VLDL (mg/d) \\
\hline HC & $45.66 \pm 0.83$ & $15.28 \pm 0.22$ & $13.56 \pm 0.19$ & $16.82 \pm 0.42$ \\
DC & $54.30 \pm 1.19$ & $11.05 \pm 0.21$ & $18.02 \pm 0.09$ & $25.23 \pm 0.89$ \\
HXT & $45.30 \pm 0.79$ & $16.38 \pm 0.14$ & $13.40 \pm 0.15$ & $16.52 \pm 0.50$ \\
DXT & $48.68 \pm 1.06$ & $16.21 \pm 0.24$ & $14.67 \pm 0.45$ & $17.80 \pm 0.37$ \\
\hline
\end{tabular}

Note that in DC mean values of TC, TG, LDL, VLDL were significantly higher $((\mathrm{P}<0.01)$ and HDL significantly lower $(\mathrm{P}<0.01)$ compared to DXT group.

\subsubsection{Serum Creatinine Level and Serum Total Protein Content}

Serum creatinine level in DC were significantly higher $((\mathrm{P}<0.01)$ as compared to all other groups. Serum total protein content in treated groups (HXT \& DXT) was significantly higher $(\mathrm{P}<0.01)$ compared to control groups $(\mathrm{HC} \& \mathrm{DC})($ Table 4$)$ [12].

Table 4. Biochemical parameters (Mean $\pm S D$ ) after co-administration of $d$ - $\alpha$-tocopherol and $d$ - $\delta$-TRF.

\begin{tabular}{lllll}
\hline Serum Analyses & & & \\
\hline Groups & Catalase $(\mathbf{u} / \mathbf{m l}) *$ & TAC $(\boldsymbol{\mu m o l} / \mathbf{L})$ & Total protein $(\mathbf{g} / \mathbf{d l})$ & Creatinine $(\mathbf{m g} / \mathbf{d l})$ \\
\hline HC & $0.0672 \pm 0.004$ & $1285.5 \pm 67.18$ & $5.05 \pm 0.07$ & $0.425 \pm 0.010$ \\
DC & $0.0438 \pm 0.005$ & $1000 \pm 67.88$ & $4.5 \pm 0.14$ & $0.790 \pm 0.022$ \\
HXT & $0.128 \pm 0.002$ & $2619 \pm 48$ & $5.47 \pm 0.15$ & $0.425 \pm 0.016$ \\
DXT & $0.081 \pm 0.001$ & $2413 \pm 27.71$ & $5.2 \pm 0.14$ & $0.545 \pm 0.018$ \\
\hline
\end{tabular}

Note that serum creatinine level was significantly high $((\mathrm{P}<0.01)$ and all other values were significantly low in DC compared to healthy control and treated groups $(\mathrm{P}<0.05)$. Catalase $(\mathrm{u} / \mathrm{ml})^{*} \mathrm{u}-\mu$ mols of $\mathrm{H}_{2} \mathrm{O}_{2}$ utilised $/ \mathrm{mt}$.

\subsubsection{Enzymatic Antioxidant and Oxidative Stress Parameter}

Serum catalase activity and total antioxidant capacity in treated groups (HXT \& DXT) exhibited significantly higher $(\mathrm{P}<0.01, \mathrm{P}<0.05)$ values compared to control groups (HC \& $\mathrm{DC})$. These analyses values in DC were significantly lower $(\mathrm{P}<0.05)$ compared to HC group (Table 4) [12].

\section{Discussion}

In diabetes increased reactive oxygen species (ROS) generation leads to myopathy due to failure of myocyte plasma membrane repair. Vitamin E promotes the myocyte plasma membrane repair and thus maintains the skeletal muscle homeostasis [4]. Myonecrosis is a complication of longstanding diabetes which most commonly affects the lower limb muscles [13].

In muscle injury the necrotic fibers are enlarged with altered internal architecture, influx of calcium ions, loss of the plasmalemma and an increased number of mononucleated cells $[14,15]$. In this current study the control groups showed more inflammatory cells, atrophic fibres with hypereosinophilic, hypertrophied and undulated sacrolemma and necrotic fibres with mineralization. Other myopathic changes such as swollen, vacuolated, hyalinized, fragmented and hemorrhagic myofibers and plethora of blood capillaries 
were noticed in diabetic control group. Three weeks coadministration of $d-\alpha$-tocopherol and $d-\delta$-TRF seems to hasten the replacement of degenerated myofibers in treated groups.

Muscle regeneration and physiological activity of stem cells are inhibited by chronic inflammatory response [14]. On $3^{\text {rd }}$ week, more cellular infiltrations were seen in the epimysium and between myofibres of control groups. In treated groups the decreased inflammations points out towards the helpful effect of $d-\alpha$-tocopherol and $d-\delta$-TRF in creating the favorable environment for muscle regrowth after injury.

Presence of split myofibers during muscle regeneration suggests incomplete fusion of fibers regenerating within the same basal lamina $[16,17]$. On $3^{\text {rd }}$ week in control groups the recently regenerated myofibers were minimal. In treated groups presence of more newly regenerated myofibers along with the split fibres revealed the efficiency of vitamin E isoforms in structural recovery of injured skeletal muscle.

Diameter of myofibers and the number of central myonuclei are two useful markers for fusion-based muscle regeneration process [18]. Reduction in the diameter of hind limb myofibres were observed in STZ-diabetic rats [19] and in the present study similar histomorphological results were observed in diabetic control group. The recently regenerated fibres are larger in diameter and have central nuclei. These myonuclei moves to the periphery at the end of muscle regeneration [20]. More number of central nuclei and increased size of muscle fibres were found in treated groups than control groups. These explain the combined effect of d$\alpha$-tocopherol and $d-\delta$-TRF on fast repair of myofibers after crushed injury.

The repair of damaged myofibers occurred by the activation, proliferation and differentiation of satellite cells into myoblasts [15]. In treated groups more myoblasts were seen as compared to control groups, suggesting thereby that both $d-\alpha$-tocopherol and $d-\delta$-TRF have potency to promote the proliferation and differentiation process of satellite cells into myoblasts.

The initial fibrosis which occurs during healing process has important role in support, strength and protection of the injury site. However, the overproduction of collagens within the injured area often leads to heavy scarring and the loss of muscular function [14]. In the present study, even on $3^{\text {rd }}$ week more fibrosis and fatty depositions in the epimyseal and perimyseal connective tissue layers were observed in control groups. Treated groups had lesser fibrosis and thus providing suitable environment for muscles regeneration evidenced as superior effects of co-administration of $d-\alpha$ tocopherol and $d-\delta$-TRF.

Extra cellular remodeling is a one of the fundamental requirements for myoblast migration and fusion during development and regeneration [21]. On $3^{\text {rd }}$ week in HC the reappearance of thin elastin fibres were observed only in epimysium while even these were absent in DC connective tissue coverings. Treated groups showed marked elastin fibres in all connective tissue coverings which is in agreement with our previous study [12] that the $d-\alpha-$ tocopherol and $d-\delta$-TRF have beneficial role in connective tissue remodeling. Regeneration process is completed only after the penetration of new blood vessels into the injured area [22]. In the present study control groups showed lesser proliferated blood capillaries in the epimysium as compared to treated groups. In addition to this treated groups had neovascularization at the site of regenerated myofibers as well. This result correlates with our many previous studies $[23,24]$ that both $d$ - $\alpha$-tocopherol and $d-\delta$-TRF supplementation supports revascularization after crushinjuries.

Reinnervation after injury is helpful for the maturation and functional recovery of regenerating myofibres. The nerve activities can directly influence protein turnover and gene expression in multinucleated regenerating myotubes and indirectly influence the proliferation and differentiation of satellite cells [25- 27]. At the end of $3^{\text {rd }}$ week the reinnervation were observed in epimysium of all groups. In treated groups the newly formed myofibers were more mature and attained functional recovery by presence of regenerated nerve bundles in relation to repaired myofibers. This result is in agreement with previous studies [23, 24] that both $\mathrm{d}$ - $\alpha$-tocopherol and $\mathrm{d}-\delta$-TRF have potency in nerve regeneration after crush injuries.

Increased levels of cholesterol, triglycerides and phospholipids are the common features in hyperlipidemia [28]. Maintaining the optimal level of lipids is a necessary requirement in diabetes to prevent the diabetic microangiopathy, macroangiopathy, cerebral vascular disease and arteriosclerosis [29]. The data of the present study indicates that the mean values of total cholesterol (TC), triglycerides (TG), low density lipoprotein (LDL) and very low density lipoprotein (VLDL) levels were higher and high density lipoprotein (HDL) level was lower in diabetic control group, indicating a state of significant dyslipidemia in untreated diabetic rats [30]. Lower mean values of TC, TG, LDL and VLDL levels and high HDL level were recorded in diabetic co-administered group (DXT) after three weeks treatment, this result is in agreement with other related study [31].

Catalase is a preventive antioxidant which inhibits the initial production of free radicals and removes the excess $\mathrm{H}_{2} \mathrm{O}_{2}$ [32]. The present study showed serum catalase activity value was lower in DC this is in agreement with other studies $[33,34]$. This activity was normalized in control group after vitamin E treatment [34]. Three weeks co-administration of $d-\alpha$-tocopherol and $d-\delta$-TRF also helped to increase the serum catalase activity in treated groups [12].

Antioxidant capacity of plasma is the primary measure and marker to evaluate the status and potential of oxidative stress in the body [35]. The present work observed that serum total antioxidant level in diabetic control was significantly lower $(\mathrm{P}<0.05)$ compared to healthy control which is in agreement with the findings of other study [36]. Improved serum antioxidant capacity was observed in treated groups after coadministration of $d-\alpha$-tocopherol and $d-\delta$-TRF for three weeks [12]. 


\section{Conclusion}

Based on all findings it is concluded that co-administration of $d-\alpha$-tocopherol and $d-\delta$-TRF have potency to maintain glycemic level, improve the antioxidant capacity and promote functional and structural recovery of myofibers after crush injuries by regeneration, revascularization, reinnervation and connective tissue remodeling. Therefore it is suggested that these vitamin $\mathrm{E}$ isoforms are hopeful option for the treatment of skeletal muscle crush-injuries in both healthy and diabetics.

\section{Acknowledgements}

All kinds of support availed from the Department of Anatomy, JN Medical College, Aligarh Muslim University is gratefully acknowledged.

\section{References}

[1] Lim JJ, Wan Ngah WZ, Mouly V, Karim NA. Reversal of Myoblast Aging By Tocotrienol Rich Fraction Post Treatment. Oxidative Medicine and Cellular Longevity 2013; 1-11.

[2] Mouly V, Aamiri A, Bigot A, Cooper RN, Di Donna S, Furling $\mathrm{D}$ et al. Themitotic clockinskeletal muscle regeneration, disease and cell mediated gene therapy. Acta Physiologica Scandinavica. 2005;184: 3-15.

[3] Aragno M, Mastrocola R, Catalano MG, Brignardello E, Danni O, Boccuzzi G. Oxidative stress impairs skeletal muscle repair in diabetic rats. Diabetes.2004; 53:1082-1088.

[4] Howard AC, McNeil AK, Paul L. Promotion of plasma membrane repair by vitamin E. Nature communications 2011; 1594: $1-8$

[5] Labazi M, McNeil AK, Kurtz T, Lee TC, Pegg RB, Angeli JPF, Conrad M, McNeil PL. The Antioxidant requirement for plasma membrane repair in skeletal muscle. Free Radical Biology and Medicine 2015; 84: 246-253.

[6] Matough FA, Budin SB, Zariyantey AH, Mariati AR, AlWahaib Ni, Jamaludine M. Tocotrienol-Rich Fraction from Palm Oil Prevents Oxidative Damage in Diabetic Rats. Sultan Qaboos University Med J 2014; 14: 95-103.

[7] Elsy B, Khan AA, Maheshwari V. Regenerative potential of d$\delta$-tocotrienol rich fraction on crushed skeletal muscle of diabetic rats. J Interdiscipl Histopathol 2017; 5(2):36-42.

[8] Elsy B, Khan AA, Maheshwari V. Effects of d- $\alpha$-Tocopherol on skeletal muscle regeneration in crushed injury of diabetic rats. Eur J Anat. 2017; 21(4): 293-304.

[9] Elsy B, Maheshwari V, Khan AA. Effects of $d \alpha$-Tocopherol on Progression of Reepithelialization, Matrix Remodeling and Appearance of Epidermal Appendages in Secondary Skin Wounds of Diabetic Rats. J Dermatolog Clin Res 2016; 4: 1081-1087.

[10] Sinha AK. Colorimetric assay of catalase. Anal Biochem 1972; 47: 389-394.

[11] Benzie IFF, Strain JJ. The ferric reducing ability of plasma (FRAP) as a measure of "antioxidant power": the FRAP assay.
Anal Biochem 1996; 239: 70-76.

[12] Elsy B, Khan AA, Maheshwari V. Effect of co-administration of vitamin $\mathrm{E}$ isoforms $\mathrm{d}-\alpha$-tocopherol and $\mathrm{d}$ - $\delta$-tocotrienol rich fraction on the healing of skin wounds in diabetic rats. Int $\mathrm{J}$ Biomed Eng Clin Sci. 2017; 3(5): 52-62.

[13] Glauser SR, Glauser J, Hatem SF. Diabetic muscle infarction: a rare complication of advanced diabetes mellitus. Emerg Radiol 2008; 15: 61-5.

[14] Musarò A. The Basis of Muscle Regeneration. Advances in Biology 2014; 612471: 1-16.

[15] Karalaki M, Fili S, Philippou A, Koutsilieris M. Muscle Regeneration: Cellular and Molecular Events. In vivo. 2009; 23: 779-796.

[16] Blaveri K, Heslop L, Yu DS, Rosenblatt JD, Gross JG, Partridge TA, Morgan JE: Patterns of repair of dystrophic mouse muscle: studies on isolated fibers. Dev Dyn 1999; 216: 244-256.

[17] Bourke DL, Ontell M. Branched myofibers in long-term whole muscle transplants: a quantitative study. Anat Rec 1984; 209: 281-288.

[18] Chargé SB, Rudnicki MA. Cellular and molecular regulation of muscle regeneration. Physiol Rev 2004; 84: 209-238.

[19] Aughsteen AA, Khair AB, Suleiman AA. Quantitative Morphometric Study of the Skeletal Muscles of Normal and Streptozotocin-Diabetic Rats. JOP J Pancreas (Online) 2006; 7:382-389.

[20] Yin H, Price F, Rudnick MA. Satellite Cells and the Muscle Stem Cell Niche. Physiol Rev 2013; 93: 23-67.

[21] Lewis MP, Tippett HL, Sinanan AC, Morgan MJ, Hunt NP. Gelatinase-B (matrix metalloproteinase-9; MMP-9) secretion is involved in the migratory phase of human and murine muscle cell cultures. J Muscle Res Cell Motil 2000; 21: 22323.

[22] Carlson BM, Faulkner JA. The regeneration of skeletal muscle fibers following injury: a review. Med Sci Sports Exerc 1983; 15: $187-198$.

[23] Elsy B, Khan AA, Maheshwari V. Neuroregenerative effects of $d$ - $\alpha$-tocopherol on the crushed sciatic nerve of diabetic rats. Innovare J Health Sci 2017; 5: 1-6.

[24] Elsy B, Khan AA, Maheshwari V. Neuroprotective effects of $\mathrm{d}$ - $\delta$-tocotrienol rich fraction on crushed sciatic nerve in diabetic rats. Eur j pharmaceutic med res, 2017; 4: 489-500.

[25] Mozdziak PE, Pulvermacher PM, Schultz E. Muscle regeneration during hind limb unloading results in a reduction in muscle size after reloading. J Appl Physiol 2001; 91:183190 .

[26] Mitchell PO, Pavlath GK. Skeletal muscle atrophy leads to loss and dysfunction of muscle precursor cells. Am J Physiol Cell Physiol 2004; 287: 1753-62.

[27] Slater CR, Schiaffino S. Skeletal muscle repair and regeneration, in Advances in Muscle Research. Springer, Amsterdam, 2008; Ed: 303-334.

[28] Bagdade JD, Helve E, Taskinen MR. Effect of continuous insulin infusion therapy lipoprotein surface and core lipid composition in IDDM. Metab. 1991; 40:445-9. 
[29] Arora P, Jain B, Kohli GK, Sehgal M, Goyal RK. Dyslipidemia in type 2 diabetes mellitus. Ind Med Gaz. 2002: 258-60.

[30] Tavaresde Almeida DA, Pereira Braga C, Barbosa Novelli EL, Henrique Fernandes AA. Evaluation of Lipid Profile and Oxidative Stress in STZ Induced Rats Treated with Antioxidant Vitamin. Braz Arch Biol Technol. 2012;55: 52736

[31] Budin SB, Othman F, Louis SR, Bakar MA, Das S and Mohamed J. The effects of palm oil tocotrienol-rich fraction supplementation on biochemical parameters, oxidative stress and the vascular wall of streptozotocin-induced diabetic rats. Clinics Sao Paulo, 64, 2009, 235-244.

[32] Vasudevan DM, Sreekumari S. Textbook of Biochemistry (For Medical Students), $4^{\text {th }}$ ed. $2005 ; 340-341$
[33] Jeyashanthi N, Ashok V. Anti-Oxidative Effect of Cassia auriculata on Streptozotocin Induced Diabetic Rats. Ind J Clin Biochem 2010; 25: 429-434.

[34] Shirpoor A, Ansari MHK, Salami S, Pakdel FG, RasmiY. Effect of vitamin $\mathrm{E}$ on oxidative stress status in small intestine of diabetic rat. World J Gastroenterol 2007; 13: 4340-4344.

[35] Tiwari BK, Pandey KB, Abidi AB, Rizvi SI. Markers of Oxidative Stress during Diabetes Mellitus. J Biomark 2013; ID 378790 .

[36] Alireza N, Bokaeian M, Mohsen S, Ali F, Azim A. Attenuation of oxidative stress in streptozotocin-induced diabetic rats by eucalyptus globulus. Ind J Clin Biochem 2009; 24: 419-425. 